\title{
Evaluating early response of cervical cancer under concurrent chemo-radiotherapy by intravoxel incoherent motion MR imaging
}

\author{
$\mathrm{Li} \mathrm{Zhu}^{1+}{ }^{\dagger}$ Lijing Zhu ${ }^{2+}$, Hua Shi ${ }^{1}$, Huanhuan Wang ${ }^{1}$, Jing Yan², Baorui Liu², Weibo Chen ${ }^{3}$, Jian He ${ }^{1 *}$, \\ Zhengyang Zhou ${ }^{*}$, Xiaofeng Yang ${ }^{4}$ and Tian Liu ${ }^{4}$
}

\begin{abstract}
Background: Intravoxel incoherent motion (IVIM) MR imaging has been applied in researches of various diseases, however its potential in cervical cancer patients has not been fully explored. The purpose of this study was to investigate the feasibility of IVIM MR imaging to monitor early treatment response in patients receiving concurrent chemo-radiotherapy (CCRT) for advanced cervical cancers.

Methods: Twenty-one patients receiving CCRT for advanced cervical cancer were prospectively enrolled. MR examinations including IVIM imaging (with 14 b values, $0 \sim 1000 \mathrm{~s} / \mathrm{mm}^{2}$ ) were performed at 4 time points: 1-week prior to, 2-week and 4-week during, as well as immediately post CCRT (within 1 week). The apparent diffusion coefficient (ADC) maps were derived from the mono-exponential model, while the diffusion coefficient (D), perfusion fraction ( $f$ ) and pseudo-diffusion coefficient $\left(D^{*}\right)$ maps were calculated from the bi-exponential model. Dynamic changes of ADC, $D, f$ and $D^{*}$ in cervical cancers were investigated as early surrogate markers for treatment response.
\end{abstract}

Results: ADC and D values increased throughout the CCRT course. Both $f$ and $D^{*}$ increased in the first 2 to 3 weeks of CCRT and started to decrease around 4 weeks of CCRT. Significant increase of $f$ value was observed from prior to CCRT $\left(f_{1}=0.12 \pm 0.52\right)$ to two-week during CCRT $\left(f_{2}=0.20 \pm 0.90, p=0.002\right)$.

Conclusions: IVIM MR imaging has the potential in monitoring early tumor response induced by CCRT in patients with cervical cancers.

Keywords: Intravoxel incoherent motion MR imaging, Tumor response, Magnetic resonance imaging, Cervical cancer, Concurrent chemo-radiotherapy

\section{Background}

Cervical cancer is the third most common malignancy in women worldwide, accounting for $9 \%$ of the total new female cancer cases [1]. Primary treatment selection is guided by tumor stage [2]. For those who are diagnosed at the locally advanced stage, concurrent chemo-radiotherapy (CCRT) is currently the standard care [3, 4]. Because ineffective treatment is associated with increased toxicity and morbidity, accelerated tumor growth, a delay in commencing alternative, potentially effective treatment, and

\footnotetext{
*Correspondence: hjxueren@126.com; zyzhou@nju.edu.cn

${ }^{\dagger}$ Equal contributors

'Department of Radiology, Nanjing Drum Tower Hospital, the Affiliated Hospital of Nanjing University Medical School, Nanjing 210008, China Full list of author information is available at the end of the article
}

unnecessary expense [5], the ability to rapidly and accurately predict the response of a tumor to therapy would have immense value in clinical practice. Therefore, studies of reliable early surrogate markers of tumor response to these cancer therapies are warranted.

The past decade has witnessed rapid developments in magnetic resonance imaging (MRI). A number of studies have established MRI as the most effective imaging modality for the diagnosis of the cervical cancers as well as the assessment of tumor response to therapy $[6,7]$. Conventional T1-weighted and T2-weighted MRIs offer anatomical information such as tumor size, while newer sequences such as perfusion-weighted (PW), diffusionweighted (DW) MRIs and MR spectroscopy (MRS) have demonstrated potential as early predictors by offering a 
combination of morphological, physiological and metabolic information. DW-MRI allows creation of image contrast based on thermally driven motion of water molecules. It is well-established that apparent diffusion coefficient (ADC) values of malignant tumors are commonly lower than those of normal tissues or benign lesions. In addition, effective anti-cancer treatment can be reflected by increased $\mathrm{ADC}$ values due to alterations of tumor cellularity and integrity of cell membrane caused by necrosis and apoptosis [7-10]. A prospective study on cervical cancer showed that the post treatment ADC values of the complete response (CR) increased rapidly compared with the partial response (PR) and stable disease (SD) groups [11]. This study further demonstrated that tumor with low pretreatment ADC values tended to respond better to CCRT than those with high baseline ADC values. A similar finding was reported in a rectal cancer study by Hein et al. [12]. These studies have indicated DWI's potential for predicting and monitoring treatment response. However, in vivo microscopic motion of water molecules is influenced by not only diffusion of water molecules, but also microcirculation of blood in the capillary network [13]. As a result, ADC values represent a combined signal of microscopic perfusion and diffusion instead of the pure diffusion.

PW-MRI provides information regarding the perfusion and permeability of tumors with time-dependent intravenous delivery of exogenous contrast agent. Dynamic contrast-enhanced MRI (DCE-MRI) has been shown useful in tumor detection as cervical tumors typically enhance intensely and early (30s) after gadolinium injection [14]. Using DCE-MRI to assess tumor response has also been reported in numerous studies [15-18], and tumors with high perfusion before therapy or increased signal intensity within the first 2 weeks after treatment appeared to indicate favorable prognosis [19]. However, the performance of DCE-MRI is more complex than other imaging techniques because the dependence of intravascular contrast media use. The unavoidable measurement error, the complicated analysis and presentation of imaging data, and potential development of nephrogenic systemic fibrosis associated with gadoliniumcontaining contrast material use also limited the clinical use of DCE-MRI [20].

Intravoxel incoherent motion (IVIM), initially described by Le Bihan et al. [21], was proposed as an extension of DW-MRI by using an increased number of $b$ values [22]. At low $b$ values, data obtained are dominated by perfusion effects, while signal delay captured at high $b$ values is mainly attributed to diffusion [23]. All these features make separate analysis of pure diffusion coefficient (D) and perfusion-related incoherent microcirculation ( $\left.D^{*}\right)$ possible. In recent years, with the development in MR hardware, a renewed interest in IVIM has been shown on various organs such as head and neck [24], prostate [25], breast [26], and kidney [27]. In these studies, IVIM offered information on both tissue characterization and tumor response.

Tumor differentiation based on the IVIM model has also been demonstrated in cervical cancer with low perfusion and diffusion characteristics [28], and IVIM parameters could discriminate cervical cancer from benign tissue. However, applications of IVIM on monitoring tumor response of cervical cancer have not been reported yet. The main purpose of this study is to evaluate the feasibility of IVIM for predicting the therapeutic efficacy of treatments in cervical cancer and to investigate IVIM as an early imaging biomarker for treatment response.

\section{Methods}

Patient and treatment characteristics

This prospective study was approved by the Committee on Medical Ethics of Nanjing Drum Tower Hospital, and all patients enrolled signed the informed consent forms. The study inclusion criteria were: 1) women with advanced cervical cancers (i.e., clinically staged IIB to IVA based on the International Federation of Gynecology and Obstetrics (FIGO) classification) diagnosed with biopsies, 2) age older than 18 years, and 3) no prior history of cervical cancer treatment. The study exclusion criteria were: 1) patients ineligible for CCRT, such as those with pregnancy, renal or liver failure, current infection and certain drug allergies and 2) patients with MRI contraindication such as pacemaker, metal implantation and claustrophobia disorder.

All patients underwent CCRT with external beam radiotherapy (EBRT) at $1.8 \sim 2.0$ Gy daily to a dose of $45 \sim 50 \mathrm{~Gy}$. The volume of the EBRT depended on the nodal status as determined by radiography before the therapy. Brachytherapy was used to boost with an additional $30 \sim 40$ Gy to point A (corresponding to the paracervical triangle in the medial edge of the broad ligament where the uterine vessels cross the ureter). Chemotherapy consisting of weekly nedaplatin or biweekly nedaplatin plus paclitaxel/docetaxel was given concomitantly with EBRT. The therapy would be stopped if the leukocytes count dropped below $3000 / \mathrm{mm}^{3}$, or the platelet count dropped below $80,000 / \mathrm{mm}^{3}$, and it was resumed once the counts rose above the levels. The duration of the chemotherapy was no more than 6 weeks, and the selection of therapeutic regimen was decided individually according to baseline health condition, tumor extent, lymph node or adjacent organ involvement.

\section{Magnetic resonance imaging}

MR examinations were performed at 4 time points: one week prior to CCRT, at the end of the 2nd week, at the end of the 4th week during CCRT, and immediately post 
CCRT (within 1 week). All MR examinations were performed with a 3.0-T MRI scanner (Achieva 3.0 T, Philips Healthcare, Best, the Netherlands) with a 16-channel torso phased-array body coil. A MRI scanning protocol was developed and used for all scans in this study. Patients were asked to take clyster $2 \sim 3 \mathrm{~h}$ before the MRI in order to reduce artifact induced by gas and feces in the rectum. The standard sequences included axial T2weighted turbo spin-echo $(\mathrm{TR}=4500 \mathrm{~ms}, \mathrm{TE}=90 \mathrm{~ms}$, matrix size $=308 \times 402$, field of view $=30 \mathrm{~cm} \times 40 \mathrm{~cm}$, slice thickness $=5 \mathrm{~mm}$, intersection gap $=0.5 \mathrm{~mm}$, number of signal averages $(\mathrm{NSA})=1$ ), sagittal T2-weighted turbo spin-echo $(\mathrm{TR}=4500 \mathrm{~ms}, \mathrm{TE}=90 \mathrm{~ms}$, matrix size $=212 \times 209$, field of view $=30 \mathrm{~cm} \times 40 \mathrm{~cm}$, slice thickness $=5 \mathrm{~mm}$, intersection gap $=0.5 \mathrm{~mm}, \mathrm{NSA}=1$ ), axialT2-weighted spectral presaturation attenuated inversion recovery (SPAIR) $(\mathrm{TR}=4700 \mathrm{~ms}, \mathrm{TE}=70 \mathrm{~ms}$, matrix size $=376 \times 389$, field of view $=20 \times 20 \mathrm{~cm}$, slice thickness $=5 \mathrm{~mm}$, intersection gap $=0.5 \mathrm{~mm}, \mathrm{NSA}=1)$, sagittal T2-weighted SPAIR(TR $=4700 \mathrm{~ms}, \mathrm{TE}=70 \mathrm{~ms}$, matrix size $=256 \times 179$, field of view $=20 \times 20 \mathrm{~cm}$, slice thickness $=5 \mathrm{~mm}$, intersection gap $=0.5 \mathrm{~mm}, \mathrm{NSA}=1$ ), 3D T1-weighted turbo-field-echo contrast-enhanced acquisition $(\mathrm{TR}=3.0 \mathrm{~ms}$, $\mathrm{TE}=1.42 \mathrm{~ms}$, field of view $=$ $256 \times 194 \mathrm{~mm}$, matrix size $=30 \mathrm{~cm} \times 40 \mathrm{~cm}$, slice thickness $=1.5 \mathrm{~mm}$, intersection gap $=0 \mathrm{~mm}, \mathrm{NSA}=1$ ). Intravenous bolus injection of $0.1 \sim 0.2 \mathrm{mmol} / \mathrm{kg}$ body weight gadodiamide was performed at a rate of $3.0 \mathrm{ml} / \mathrm{s}$, followed by a $15 \mathrm{ml}$ saline flush with high pressure injector after contraindications such as severe renal failure and liver transplantation had been excluded. The scanning time of IVIM was approximately $10 \mathrm{~min}$ and the total scanning time was about $30 \mathrm{~min}$.

All the examinations were acquired with free breathing. Fourteen $b$ values $(0,10,20,30,40,50,100,150,200,350$, $500,650,800,1000 \mathrm{~s} / \mathrm{mm}^{2}$ ) were used in the axial single shot diffusion weighted echo planar imaging (SS-EPI) $(\mathrm{TR}=2834 \mathrm{~ms}, \mathrm{TE}=105 \mathrm{~ms}$, matrix size $=152 \times 120$, field of view $=30 \times 40 \mathrm{~cm}$, slice thickness $=6 \mathrm{~mm}$, intersection gap $=0.5 \mathrm{~mm}, \mathrm{NSA}=1$ ).

\section{Image and data analysis}

All MR images were independently analyzed by 2 experienced radiologists (Jian He, Zhengyang Zhou) with 6 and 8 years' experience in gynecology. The radiologists were blinded to each other's reading. The dataset was analyzed based on the bi-exponential IVIM model introduced by Le Bihan [29] with the following function: $S_{b} / S_{0}=(1-f)$ * $\exp (-b * \mathrm{D})+f * \exp \left(-b *\left(\mathrm{D}^{*}+\mathrm{D}\right)\right)$, in which $S_{b}$ represents the mean signal intensity with diffusion gradient $b, S_{0}$ represents the mean signal intensity when $b=0 \mathrm{~s} / \mathrm{mm} 2$. The IVIM data was evaluated using DWI-Tool developed by Philips with IDL 6.3 (ITT Visual Information Solutions, Boulder, $\mathrm{CO}$ ) for $\mathrm{D}, f$ and $\mathrm{D}^{*}$ maps. The tumor on MRI was defined as a mass with higher signal intensity than the adjacent cervical stroma yet lower signal intensity than the fluid signal in the urinary bladder on a T2-weighted image [30]. The specific slice of DWI with the biggest tumor section was selected referring to the corresponding axial T2-weighted images, and then, a region of interest (ROI) was manually drawn as large as possible along the inside of the tumor margin. The macroscopic necrotic areas, large vessels and areas with artifacts induced by airwater interface were excluded during the tumor contour. The longest diameter of the tumor was subsequently measured. If no residual tumor was observed after treatment, five equal-sized ROIs (each $5 \mathrm{~mm}^{2}$ ) were placed within the solid components of the tumor region prior to treatment, and the diameter of the lesion would be recorded as $0 \mathrm{~cm}$. The ADC values were derived from the monoexponential model, while the $\mathrm{D}, f$ and $\mathrm{D} *$ values were calculated with bi-exponential model. The mean values of the two radiologists' measurement were calculated as the final results. The ROIs were transferred to the corresponding IVIM parametric maps with the Image J $(\mathrm{NIH}$, Bethesda, MD, USA).

\section{Treatment outcome analysis}

According to the evaluation criteria in solid tumors (RECSIT) [31], response to treatment was decided by the shrinkage of tumor size. Tumor response was classified into four groups: (1) complete response (CR) was concluded if no residual tumor can been seen on the MRI images; (2) partial response (PR) was concluded if an over $30 \%$ size reduction of the tumor was observed as compared with the original size; (3) progress disease (PD) was concluded if there was at least $20 \%$ increase in the longest diameter of tumor in comparison with the pre-treatment size; (4) stable disease (SD) was concluded if there was neither sufficient decrease to qualify for PR nor sufficient increase to qualify for PD.

\section{Statistical analysis}

All the statistical analysis was performed using SPSS 16.0 (SPSS Inc., Chicago, IL). Significant changes of IVIM parameters with time were tested using paired $t$ test. In order to evaluate the correlations between IVIM parameters, the Pearson's correlation coefficient was calculated with $95 \%$ confidence interval. Two-tailed $p$ values were used and $p$ values less than 0.05 were considered as statistically significant. An intraclass correlation coefficient (ICC) was calculated to evaluate inter-observer reliability.

\section{Results}

From December 2013 to January 2015, 21 patients with locally advanced cervical cancers were enrolled in this prospective study, and all of them were confirmed histologically as squamous cell carcinoma. The patient and 
Table 1 Patient and treatment characteristics

\begin{tabular}{ll}
\hline Clinical features & Values \\
\hline No of patients & 21 \\
Age (years) & $49.6(24-76)$ \\
FIGO stage: & $11(52.4 \%)$ \\
II & $6(28.6 \%)$ \\
III & $4(19.0 \%)$ \\
IV & \\
Metastasis $(n=4)$ & $1(4.8 \%)$ \\
Bladder & $3(14.3 \%)$ \\
Rectum & \\
Treatment outcome & $18(85.7 \%)$ \\
Complete response & $3(14.3 \%)$ \\
Partial response &
\end{tabular}

Data are $\mathrm{N}$ (\%) or mean (range)

FIGO the International Federation of Gynecology and Obstetrics

treatment characteristics are summarized in Table 1. Eighteen of those patients were classified as CR and three as PR after the treatment. Clinical information at four time points was collected from all patients.

The mean values of diameter, $\mathrm{D}, f, \mathrm{D}^{*}$, and ADC obtained for all patients over time are shown in Table 2 . The initial mean tumor size was $4.17 \pm 1.23 \mathrm{~cm}$, and the diameter decreased significantly after treatment. All 21 patients enrolled achieved efficient local control at the end of therapy, with no one classified as PD or SD. Temporal reduction of lesions is shown on the IVIM parameter maps (Fig. 1).

All IVIM parameters showed big increases at week 2 during CCRT, values of D and ADC kept increasing during CCRT, while values of $f$ and $\mathrm{D}^{*}$ went down after week 4 during CCRT (Fig. 2). A positive correlation between $\mathrm{D}$ and ADC values was demonstrated at each time point $\left(p_{1}<0.001, p_{2}=0.003, p_{3}=0.032, p_{4}<0.001\right.$ respectively), but no significant correlations between other parameters were found in this study. While comparing the values at different time points, no significant difference was found between the ADC values at time points 3 and $4(p=0.879), f$ values at time points 2 and 3 , as well as 2 and $4\left(p_{23}=0.408, p_{24}=0.337\right)$, and among $D^{*}$ values of all time points $\left(p_{12}=0.557, p_{13}=0.461, p_{14}=\right.$ $\left.0.480, p_{23}=0.875, p_{24}=0.913, p_{34}=0.954\right)$. But all the parameters showed remarkable statistical differences between the rest time points (all $p<0.05$ ) indicating detectable changes in IVIM parameters during CCRT. The detailed results were shown in Table 3.

The ICC between two observers was 0.964 (95\% CI, $0.887 \sim 0.988 ; p<0.001)$, and the ROI area was $895.82 \pm$ $596.51 \mathrm{~mm}^{2}$ (range: $160.20 \sim 2037.45 \mathrm{~mm}^{2}$ )

\section{Discussion}

In this study, we demonstrated the potential of IVIM MR imaging in monitoring early CCRT response of cervical cancer. To the best of our knowledge, this is the first report on such an application in cervical cancer.

In cervical cancer, DWI was first reported as a diagnostic tool to distinguish the malignancy from normal uterine cervix [32]. In our study, the initial ADC and D values of cervical cancer $\left(\mathrm{ADC}=1.00 \pm 0.11 \times 10^{-3} \mathrm{~mm}^{2} / \mathrm{s}\right.$, $\left.\mathrm{D}=0.85 \pm 0.12 \times 10^{-3} \mathrm{~mm}^{2} / \mathrm{s}\right)$ were in line with the published results $\left(\mathrm{ADC}=0.99 \pm 0.18 \times 10^{-3} \mathrm{~mm}^{2} / \mathrm{s}, \mathrm{D}=\right.$ $0.86 \pm 0.16 \times 10^{-3} \mathrm{~mm}^{2} / \mathrm{s}$ ) [28] which were significantly different from normal cervical tissues $(\mathrm{ADC}=1.57 \pm$ $\left.0.17 \times 10^{-3} \mathrm{~mm}^{2} / \mathrm{s}, \mathrm{D}=1.32 \pm 0.12 \times 10^{-3} \mathrm{~mm}^{2} / \mathrm{s}\right)$. The low $\mathrm{ADC}$ and $\mathrm{D}$ values of cervical cancer were due to the increased cellularity, which was an important factor that influences the extracellular space and microscopic water diffusion in tumor tissue. In tumor tissues, the ADC values were higher than $D$ values, which suggested that a mono-exponential model could overestimate the water diffusion in the cancerous tissue, because of the "contamination" of ADC as a combined signal measurement of the microscopic perfusion and diffusion.

Previous studies $[8,33]$ have demonstrated that effective anticancer treatment would result in an increased water diffusion. Findings in this study supported the association between the ADC change and post-therapy response. Similarly, we demonstrated that the mean ADC of responders increased after treatment. D value changed with ADC value and both increased over the course of treatment. Significant differences in ADC values were demonstrated at various time points except for the last stage of therapy $(p=0.879)$. Less restricted motion of

Table 2 Changes of IVIM parameters and tumor size during the concurrent chemo- radiotherapy course

\begin{tabular}{lllll}
\hline Variables & Time point 1 & Time point 2 & Time point 3 & Time point 4 \\
\hline Diameter $(\mathrm{cm})$ & $4.17 \pm 1.23$ & $2.56 \pm 1.26$ & $1.14 \pm 1.12$ & $0.20 \pm 0.53$ \\
$f$ & $0.12 \pm 0.52$ & $0.20 \pm 0.90$ & $0.22 \pm 0.79$ & $0.18 \pm 0.58$ \\
$D^{*}\left(\times 10^{-3} \mathrm{~mm}^{2} / \mathrm{s}\right)$ & $29.23 \pm 26.49$ & $33.73 \pm 21.76$ & $34.80 \pm 21.00$ & $34.44 \pm 17.30$ \\
$A D C\left(\times 10^{-3} \mathrm{~mm}^{2} / \mathrm{s}\right)$ & $1.00 \pm 0.11$ & $1.39 \pm 0.26$ & $1.66 \pm 0.17$ & $1.67 \pm 0.16$ \\
$D\left(\times 10^{-3} \mathrm{~mm}^{2} / \mathrm{s}\right)$ & $0.85 \pm 0.12$ & $1.09 \pm 0.14$ & $1.31 \pm 0.11$ & $1.41 \pm 0.13$ \\
\hline
\end{tabular}

$\overline{A D C}$ apparent diffusion coefficient, $D$ diffusion coefficient, $f$ perfusion fraction, $D^{*}$ pseudo-diffusion coefficient 

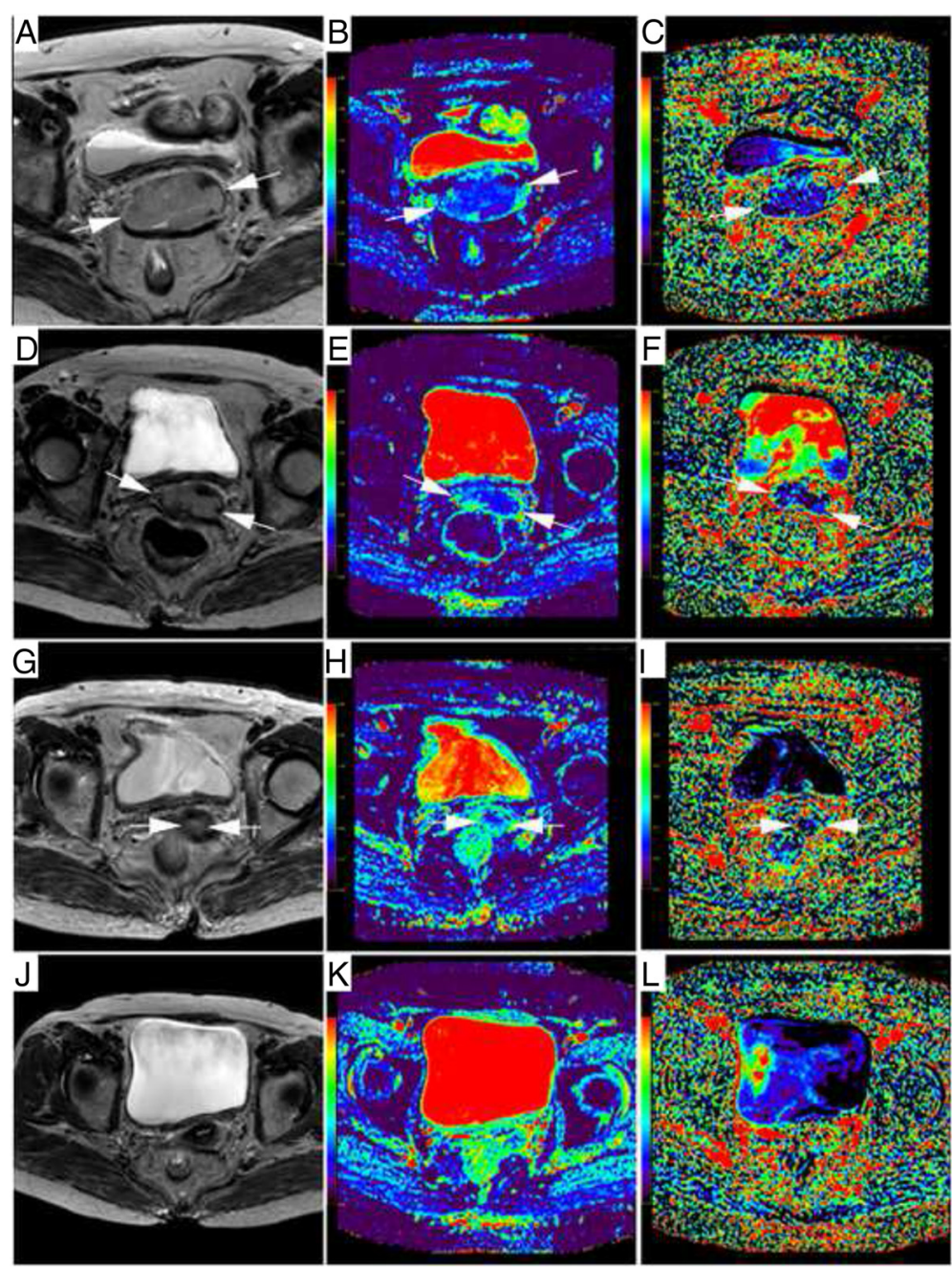

Fig. 1 MR images of a patient receiving concurrent chemo-radiotherapy (CCRT) for advanced cervical cancer (FIGO stage IIIA). a Axial T2-weighted image before CCRT shows a hyperintense mass lesion (arrows) at the cervix, with a maximal diameter of $6.0 \mathrm{~cm}$. b ADC map corresponding to (a) shows a low ADC value $\left(0.933 \times 10^{-3} \mathrm{~mm}^{2} / \mathrm{s}\right)$ of the lesion compared with the adjacent normal cervical tissues $\left(1.54 \times 10^{-3} \mathrm{~mm}^{2} / \mathrm{s}\right)$. c The $\mathrm{fmap}$ corresponding to (a) shows a low $f$ value (0.097) of the lesion compared with the adjacent normal cervical tissues (0.198); $\mathbf{d}$ Two weeks after initiation of CCRT, the diameter of cervical cancer decreased to $4.2 \mathrm{~cm}$. e The ADC value of the lesion corresponding to (d) increased to $1.038 \times 10^{-3} \mathrm{~mm} / \mathrm{s}$ compared with (b). $\mathbf{f}$ The $f$ value of the lesion corresponding to (d) increased to 0.116 compared with $(\mathbf{c})$. $\mathbf{g}$ One month after initiation of CCRT, the lesion continues to decrease with a diameter of $1.7 \mathrm{~cm}$. $\mathbf{h}$ The ADC value of the lesion corresponding to $(\mathbf{g})$ continues to increase to $1.563 \times 10^{-3} \mathrm{~mm} / \mathrm{s}$ compared to (e). $\mathbf{i}$ The $f$ value of cervical cancer corresponding to $(\mathbf{g})$ continues to increase to 0.239 compared to (f). $\mathbf{j}$ Post CCRT, no residual lesion is observed in the axial T2-weighted image. $\mathbf{k}$ The ADC value of the lesion corresponding to (j) continues to increase to $1.737 \times 10^{-3} \mathrm{~mm}^{2} / \mathrm{s} \mathrm{compared} \mathrm{to} \mathrm{(h)}$. I The $f$ value of cervical cancer corresponding to $(\mathbf{g})$ continues to increase to 0.165 compared to (i)

water molecules induced by damages in tumor tissue (e.g., loss of cell membrane integrity and a subsequent reduction in tumor cell density) after successful treatment may explain these changes [34]. These changes appeared as early as 2 weeks during CCRT, indicating the sensitivity of ADC and D as surrogate markers of early tumor response.

In this study, the pre-treatment perfusion fraction $f$ $(0.12 \pm 0.52)$ of cervical cancer was close to $0.149 \pm 0.26$ as reported by Lee EY et al. [28]. Significant higher initial $f$ values were displayed in head-and-neck cancer patients with poor clinical outcome [24]. As no patients were classified as SD or PD in this study, a further research with a larger sample size is warranted. At follow-ups, $f$ significantly increased after two-week of CCRT $(p=0.002)$ making it a potential early indicator for post-therapy response. As an imaging biomarker for the vascular compartment, the $f$ value most likely reflects the vascular volume fraction of the tumor instead of the accurate blood volume/plasma volume, it is mainly related to the regional blood flow in DCEMRI. Also by decreasing cell density and modulating the tumor microenvironment, CCRT is considered to improve the blood supply and oxygenation status of 

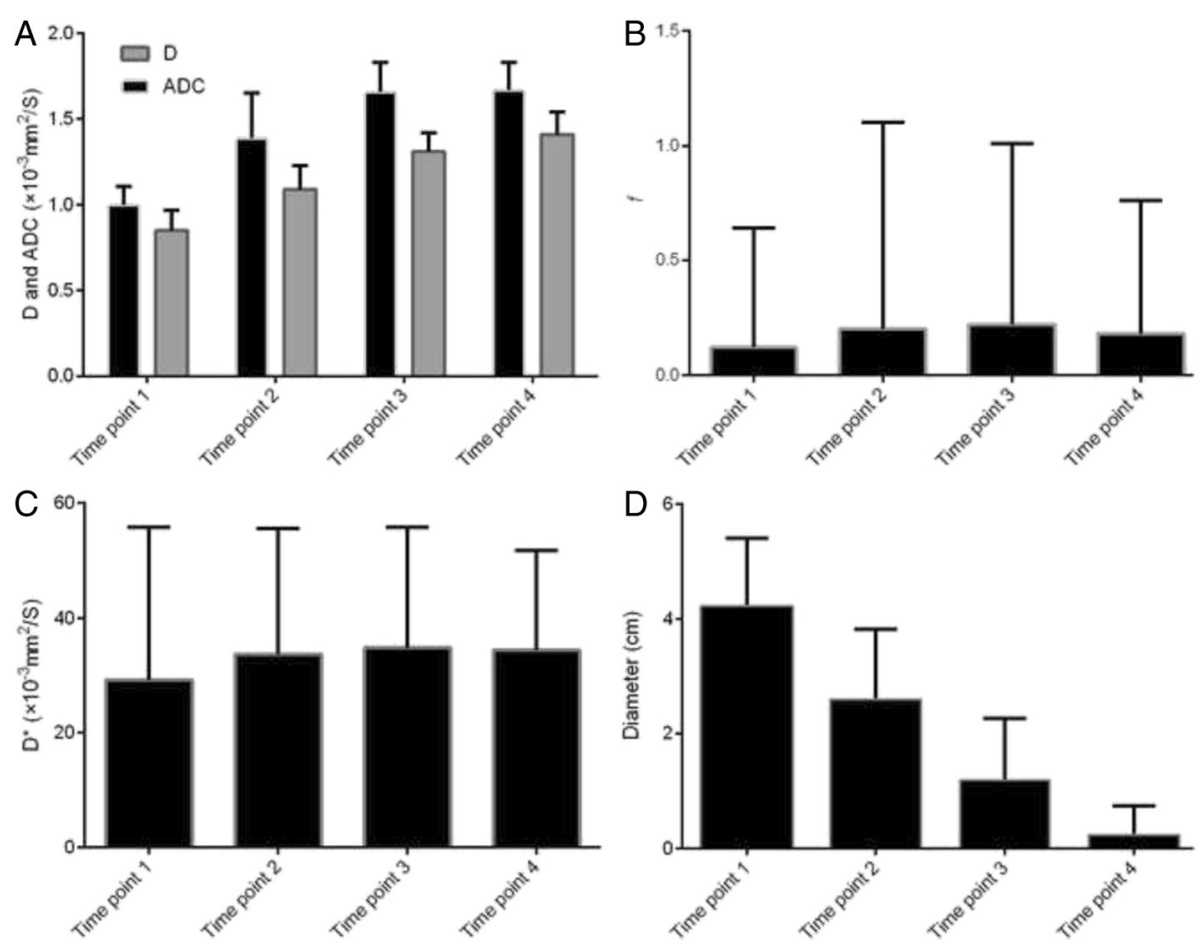

Fig. 2 Dynamic changes of various MR parameters of cervical cancers during concurrent chemo-radiotherapy (CCRT). a D and apparent diffusion coefficient (ADC) values of cervical cancers increase during the course of CCRT and the D values are lower than the ADC values. $\mathbf{b} f$ values of cervical cancers increases initially and decreases after 4 weeks of therapy. $\mathbf{c} D^{*}$ values of cervical cancers share the same tendency as $f$. $\mathbf{d}$ Tumor sizes shrink over the course of CCRT. Time point 1, before CCRT within one week; time point 2, at the end of the second week of CCRT; time point 3 , at the end of the first month of CCRT; time point 4, immediately after CCRT within one week

tumor cells, resulting in a substantial reduction of radiation-resistant hypoxic tumor cells [35]. Therefore the tumor perfusion is associated with the effect of CCRT. Although Shibuya $K$ et al [36] have shown that blood flow increased after 20 Gy of radiation therapy in cervical cancer, Ganten M K et al [37] and Gaeta $M$ et al [38] found no significant changes of $f$ over time in rectal cancers. This contradiction may be explained by different histopathological features and capillary network distribution in different types of tumors. Moreover, such discrepancy could also be caused by different imaging techniques and treatment regimes in the clinical studies.
During CCRT, the $f$ initially increased and later decreased around 4 weeks into therapy. This decline may be explained by the hypothesis that: local fibrosis and angiolysis (vessel occlusion, reduction, or disappearance) consequent on the large cumulative radiation dose altered the microcirculation in various ways, and replaced the leading role of cell lysis in the early period, and the perfusion changes comprise a greater percentage as a result. Previous study in lung has shown the role of various early inflammatory proteins in stimulating proliferation and migration myofibroblasts during later fibrosis [39]. As most patients finished their EBRT at the 5th week, transformation of therapy regimen may be answerable to the

Table 3 Differences of IVIM parameters between different time points in locally advanced cervical cancer patients under concurrent chemo- radiotherapy (CCRT)

\begin{tabular}{|c|c|c|c|c|c|c|}
\hline Variables & Time point 1 versus 2 & Time point 1 versus 3 & Time point 1 versus 4 & Time point 2 versus 3 & Time point 2 versus 4 & $\overline{\text { Time point } 3 \text { versus } 4}$ \\
\hline Diameter & $<0.001$ & $<0.001$ & $<0.001$ & $<0.001$ & $<0.001$ & $<0.001$ \\
\hline $\mathrm{D}$ & $<0.001$ & $<0.001$ & $<0.001$ & $<0.001$ & $<0.001$ & 0.016 \\
\hline$A D C$ & $<0.001$ & $<0.001$ & $<0.001$ & $<0.001$ & $<0.001$ & 0.879 \\
\hline$f$ & 0.002 & $<0.001$ & 0.005 & 0.408 & 0.337 & 0.047 \\
\hline$D^{*}$ & 0.557 & 0.461 & 0.480 & 0.875 & 0.913 & 0.954 \\
\hline
\end{tabular}

Data are shown as $p$ value from each comparison. Time point 1 , before CCRT within one week; time point 2 , at the end of the second week of CCRT; time point 3 , at the end of the first month of CCRT; time point 4, immediately after CCRT within one week

$A D C$ apparent diffusion coefficient, $D$ diffusion coefficient, $f$ perfusion fraction, $D^{*}$ pseudo-diffusion coefficient 
alteration between time point 3 and 4, whereas further histopathological examinations are needed for verification.

In tumors, $\mathrm{D}^{*}$ reflects the rate of microcapillary blood flow and is positively related to $\mathrm{f}$ according to the double exponential model theory. Our study supported this observation by showing a changing $\mathrm{D}^{*}$ in agreement with $f$. As $\mathrm{D}^{*}$ value is well known for its huge standard deviation, data instability and its dependence on signal-tonoise ratio (SNR), wider clinical application is limited in this situation. A recent IVIM imaging study of hepatocellular carcinoma has shown poor reproducibility of $\mathrm{D}^{*}$ with a mean coefficient of variation equal to $60.6 \%$ [40]. Once further improvement is achieved to the robustness and reproducibility of $\mathrm{D}^{*}$, it might be suitable to bring it into estimating the tumor response.

There are several limitations in this study. First is the relatively small sample size of patients, only 21 patients were involved in this prospective study. What's more, all patients enrolled were histologically confirmed as squamous cell carcinoma without adenocarcinoma or other types of carcinoma. Most participants in our study were staged as II (52.4\%), patients clinically classified as III or IV is relatively few. All these factors made our sample lack of representative, a source where bias may occur. Nevertheless, the preliminary results were promising and further studies with a larger and more diverse cohort are warranted. A second limitation of this study was that the appropriate number of $b$ values suitable for cervix is still unknown. Various numbers were used in the previous studies and we used $14 b$ values in this study. So searching for the best selection of $b$ values in cervix imaging would be another optimization strategy in further studies. Thirdly, without histological confirmation MRI could be inaccurate at times. For instance, a residual tumor and post-treatment fibrosis may be difficult to differentiate. Since multiple biopsies are impractical, future animal experiments could help us better understand early tumor response.

\section{Conclusions}

IVIM MR imaging has shown dynamic changes of cervical cancers during treatment, which makes IVIM parameters as potential biomarkers for tumor response following cervical cancer CCRT. Clinical studies with a large cohort to confirm these promising results are warranted. With technological advances, IVIM will become a valuable imaging tool in the clinic as well as in cancer research.

\footnotetext{
Abbreviations

ADC: apparent diffusion coefficient; CCRT: concurrent chemo-radiotherapy; CR: complete response; D: diffusion coefficient; $D^{*}$ : pseudo-diffusion coefficient; DCE: dynamic contrast-enhanced; DW: diffusion-weighted; EBRT: external beam radiotherapy; $f$; perfusion fraction; FIGO: international federation of gynecology and obstetrics; IVIM: intravoxel incoherent motion; MRI: magnetic resonance imaging; NSA: number of signal averages;
}

PD: progress disease; PR: partial response; ROC: receiver operating characteristics; ROI: region of interest; SD: stable disease; SD: standard deviation; SNR: signal-to-noise ratio; SPAIR: spectral presaturation attenuated inversion recovery; SS-EPI: single shot diffusion weighted echo planar imaging.

\section{Competing interests}

The authors declare that they have no competing interests.

\section{Authors' contributions}

LZ and HS carried out the data analysis and drafted the manuscript; LJZ and HHW had significant roles in the data acquisition; LZ and LZZ performed the whole revision; JY and BRL are the oncologists responsible for all oncological support; WBC carried out the quality control of data and algorithms; XFY and TL had significant roles in the study design and manuscript review; $\mathrm{JH}$ and $\mathrm{ZYZ}$ formulated the research question, supervised the research program and edited the manuscript. All authors read and approved the final manuscript.

\section{Acknowledgments}

This work was supported by grants from National Natural Science Foundation of China $(81371516,81501441)$, Natural Science Foundation of Jiangsu Province (BK20150109), Six talent peaks project of Jiangsu Province (2015-WSN-079) and Jiangsu Provincial Health and Family Planning Commission Youth Scientific Research Project (Q201508).

\section{Author details}

'Department of Radiology, Nanjing Drum Tower Hospital, the Affiliated Hospital of Nanjing University Medical School, Nanjing 210008, China. ${ }^{2}$ The Comprehensive Cancer Centre of Drum Tower Hospital, the Affiliated Hospital of Nanjing University Medical School, Nanjing 210008, China. ${ }^{3}$ Philips Healthcare, Shanghai, China. ${ }^{4}$ Department of Radiation Oncology and Winship Cancer Institute, Emory University, Atlanta, GA 30322, USA.

Received: 23 August 2015 Accepted: 3 February 2016

Published online: 10 February 2016

\section{References}

1. Jemal A, Bray F, Center MM, Ferlay J, Ward E, Forman D. Global cancer statistics. CA Cancer J Clin. 2011:61(2):69-90.

2. Belhadj H, Berek J, Bermudez A, Bhatla N, Cain J, Denny L, et al. FIGO staging for carcinoma of the vulva, cervix, and corpus uteri. Int J Gynaecol Obstet. 2014;125(2):97-8.

3. Rose PG, Bundy BN, Watkins EB, Thigpen JT, Deppe G, Maiman MA, et al. Concurrent cisplatin-based radiotherapy and chemotherapy for locally advanced cervical cancer. N Engl J Med. 1999;340(15):1144-53.

4. Whitney CW, Sause W, Bundy BN, Malfetano JH, Hannigan EV, Fowler WJ, et al. Randomized comparison of fluorouracil plus cisplatin versus hydroxyurea as an adjunct to radiation therapy in stage IIB-IVA carcinoma of the cervix with negative para-aortic lymph nodes: a Gynecologic Oncology Group and Southwest Oncology Group study. J Clin Oncol. 1999;17(5):1339-48.

5. Kirwan JM, Symonds P, Green JA, Tierney J, Collingwood M, Williams CJ. A systematic review of acute and late toxicity of concomitant chemoradiation for cervical cancer. Radiother Oncol. 2003;68(3):217-26.

6. Harry VN, Semple SI, Parkin DE, Gilbert FJ. Use of new imaging techniques to predict tumour response to therapy. Lancet Oncol. 2010;11(1):92-102.

7. Kundu S, Chopra S, Verma A, Mahantshetty U, Engineer R, Shrivastava SK. Functional magnetic resonance imaging in cervical cancer: current evidence and future directions. J Cancer Res Ther. 2012;8(1):11-8.

8. Pickles MD, Gibbs P, Lowry M, Turnbull LW. Diffusion changes precede size reduction in neoadjuvant treatment of breast cancer. Magn Reson Imaging. 2006;24(7):843-7.

9. Kim S, Loevner L, Quon H, Sherman E, Weinstein G, Kilger A, et al. Diffusionweighted magnetic resonance imaging for predicting and detecting early response to chemoradiation therapy of squamous cell carcinomas of the head and neck. Clin Cancer Res. 2009;15(3):986-94.

10. Kim HS, Kim CK, Park BK, Huh SJ, Kim B. Evaluation of therapeutic response to concurrent chemoradiotherapy in patients with cervical cancer using diffusion-weighted MR imaging. J Magn Reson Imaging. 2013;37(1):187-93.

11. Liu Y, Bai R, Sun H, Liu H, Zhao X, Li Y. Diffusion-weighted imaging in predicting and monitoring the response of uterine cervical cancer to combined chemoradiation. Clin Radiol. 2009;64(11):1067-74. 
12. Hein PA, Kremser C, Judmaier W, Griebel J, Rudisch A, Pfeiffer KP, et al. [Diffusion-weighted MRI-a new parameter for advanced rectal carcinoma?]. Röfo. 2003;175(3):381-6.

13. Mazaheri Y, Afaq A, Rowe DB, Lu Y, Shukla-Dave A, Grover J. Diffusionweighted magnetic resonance imaging of the prostate: improved robustness with stretched exponential modeling. J Comput Assist Tomogr. 2012;36(6):695-703.

14. Thomassin-Naggara I, Siles P, Balvay D, Cuenod CA, Carette MF Bazot M. MR perfusion for pelvic female imaging. Diagn Interv Imaging 2013;94(12):1291-8

15. Yuh WT, Mayr NA, Jarjoura D, Wu D, Grecula JC, Lo SS, et al. Predicting control of primary tumor and survival by DCE MRI during early therapy in cervical cancer. Invest Radiol. 2009;44(6):343-50.

16. Andersen EK, Hole KH, Lund KV, Sundfor K, Kristensen GB, Lyng H, et al. Pharmacokinetic parameters derived from dynamic contrast enhanced MR of cervical cancers predict chemoradiotherapy outcome. Radiother Oncol. 2013;107(1):117-22.

17. Andersen EK, Kristensen GB, Lyng H, Malinen E. Pharmacokinetic analysis and $\mathrm{k}$-means clustering of DCEMR images for radiotherapy outcome prediction of advanced cervical cancers. Acta Oncol. 2011;50(6):859-65.

18. Park JJ, Kim CK, Park SY, Simonetti AW, Kim E, Park BK, et al. Assessment of early response to concurrent chemoradiotherapy in cervical cancer: value of diffusion-weighted and dynamic contrast-enhanced MR imaging. Magn Reson Imaging. 2014;32(8):993-1000.

19. Gong QY, Brunt JN, Romaniuk CS, Oakley JP, Tan LT, Roberts N, et al. Contrast enhanced dynamic MRI of cervical carcinoma during radiotherapy: early prediction of tumour regression rate. Br J Radiol. 1999:72(864):1177-84.

20. High WA, Ayers RA, Chandler J, Zito G, Cowper SE. Gadolinium is detectable within the tissue of patients with nephrogenic systemic fibrosis. J Am Acad Dermatol. 2007:56(1):21-6.

21. Le Bihan D, Breton E, Lallemand D, Grenier P, Cabanis E, Laval-Jeantet M. MR imaging of intravoxel incoherent motions: application to diffusion and perfusion in neurologic disorders. Radiology. 1986;161(2):401-7.

22. Koh DM, Collins DJ, Orton MR. Intravoxel incoherent motion in body diffusion-weighted MRI: reality and challenges. AJR Am J Roentgenol. 2011; 196(6):1351-61.

23. Padhani AR, Liu G, Koh DM, Chenevert TL, Thoeny HC, Takahara T, et al. Diffusion-weighted magnetic resonance imaging as a cancer biomarker: consensus and recommendations. Neoplasia (New York, NY). 2009;11(2):102-25.

24. Hauser $T$, Essig M, Jensen A, Gerigk L, Laun FB, Munter $M$, et al. Characterization and therapy monitoring of head and neck carcinomas using diffusion-imaging-based intravoxel incoherent motion parameterspreliminary results. Neuroradiology. 2013;55(5):527-36.

25. Shinmoto H, Tamura C, Soga S, Shiomi E, Yoshihara N, Kaji T, et al. An intravoxel incoherent motion diffusion-weighted imaging study of prostate cancer. AJR Am J Roentgenol. 2012;199(4):W496-500.

26. Liu C, Liang C, Liu Z, Zhang S, Huang B. Intravoxel incoherent motion (IVIM) in evaluation of breast lesions: comparison with conventional DWI. Eur J Radiol. 2013:82(12):e782-9.

27. Rheinheimer S, Schneider F, Stieltjes B, Morath C, Zeier M, Kauczor HU, et al. IVIM-DWI of transplanted kidneys: reduced diffusion and perfusion dependent on cold ischemia time. Eur J Radiol. 2012;81(9):e951-6.

28. Lee EYP, Yu X, Chu MMY, Ngan HYS, Siu SWK, Soong IS, et al. Perfusion and diffusion characteristics of cervical cancer based on intraxovel incoherent motion MR imaging-a pilot study. Eur Radiol. 2014;24(7):1506-13.

29. Le Bihan D. BELD: Separation of diffuseion and perfusion in intravoxel incoherent motion MR imaging. Radiology. 1988:168:497-505.

30. Hatano K, Sekiya Y, Araki H, Sakai M, Togawa T, Narita Y, et al. Evaluation of the therapeutic effect of radiotherapy on cervical cancer using magnetic resonance imaging. Int J Radiat Oncol Biol Phys. 1999;45(3):639-44.

31. Watanabe H, Okada M, Kaji Y, Satouchi M, Sato Y, Yamabe Y, et al. [New response evaluation criteria in solid tumours-revised RECIST guideline (version 1.1)]. Gan to kagaku ryoho. Cancer Chemother. 2009;36(13):2495-501.

32. Naganawa S, Sato C, Kumada H, Ishigaki T, Miura S, Takizawa O. Apparent diffusion coefficient in cervical cancer of the uterus: comparison with the normal uterine cervix. Eur Radiol. 2005;15(1):71-8.

33. Moffat BA, Chenevert TL, Lawrence TS, Meyer CR, Johnson TD, Dong Q, et al. Functional diffusion map: a noninvasive MR1 biomarker for early stratification of clinical brain tumor response. Proc Natl Acad Sci U S A. 2005:102(15):5524-9.
34. Ross BD, Moffat BA, Lawrence TS, Mukherji SK, Gebarski SS, Quint DJ, et al. Evaluation of cancer therapy using diffusion magnetic resonance imaging. Mol Cancer Ther. 2003;2(6):581-7.

35. Lyng H, Sundfor K, Trope C, Rofstad EK. Disease control of uterine cervical cancer: relationships to tumor oxygen tension, vascular density, cell density, and frequency of mitosis and apoptosis measured before treatment and during radiotherapy. Clin Cancer Res. 2000;6(3):1104-12.

36. Shibuya K, Tsushima Y, Horisoko E, Noda SE, Taketomi-Takahashi A, Ohno T, et al. Blood flow change quantification in cervical cancer before and during radiation therapy using perfusion CT. J Radiat Res. 2011;52(6):804-11.

37. Ganten MK, Schuessler M, Bauerle T, Muenter M, Schlemmer HP, Jensen A et al. The role of perfusion effects in monitoring of chemoradiotherapy of rectal carcinoma using diffusion-weighted imaging, vol. 13. 2013. p. 548-56

38. Gaeta M, Benedetto C, Minutoli F, D'Angelo T, Amato E, Mazziotti S, et al. Use of diffusion-weighted, intravoxel incoherent motion, and dynamic contrast-enhanced MR imaging in the assessment of response to radiotherapy of lytic bone metastases from breast cancer. Acad Radiol. 2014;21(10):1286-93.

39. Tsoutsou PG, Koukourakis MI. Radiation pneumonitis and fibrosis: mechanisms underlying its pathogenesis and implications for future research. Int J Radiat Oncol Biol Phys. 2006;66(5):1281-93.

40. Kakite S, Dyvorne H, Besa C, Cooper N, Facciuto M, Donnerhack C, et al. Hepatocellular carcinoma: short-term reproducibility of apparent diffusion coefficient and intravoxel incoherent motion parameters at 3.0T. J Magn Reson Imaging. 2015;41(1):149-56.

\section{Submit your next manuscript to BioMed Central and we will help you at every step:}

- We accept pre-submission inquiries

- Our selector tool helps you to find the most relevant journal

- We provide round the clock customer support

- Convenient online submission

- Thorough peer review

- Inclusion in PubMed and all major indexing services

- Maximum visibility for your research

Submit your manuscript at www.biomedcentral.com/submit 